\title{
THE GENERALIZED CROFOOT TRANSFORM
}

\author{
REWAYAT KHAN
}

Abstract. We introduce a generalized Crofoot transform between the model spaces corresponding to matrix-valued inner functions. As an application, we obtain results about matrix-valued truncated Toeplitz operators.

Mathematics subject classification (2010): Primary 47B35, 47A45; Secondary 47B32, 30 J05.

Keywords and phrases: Generalized Crofoot tranform, conjugation, matrix valued truncated Toeplitz operators.

\section{REFERENCES}

[1] J. A. BALL, A. Lubin, On a class of contractive perturbations of restricted shifts, Pacific J. Math. 63 (1976), 309-323.

[2] Ch. Benhida, D. Timotin, Functional models and finite dimensional perturbations of the shift, Integral Equ. Oper. Theory 29 (1997), 187-196.

[3] Ch. Benhida, D. Timotin, Finite rank perturbations of contractions, Integral Equ. Oper. Theory 36 (2000), 253-268.

[4] N. Chevrot, E. Fricain, D. Timotin, The characteristic function of a complex symmetric contraction, Proc. Amer. Math. Soc. 135 (2007), 2877-2886.

[5] J. A. Cima, S. R. Garcia, W. T. Ross, W. R Wogen, Truncated Toeplitz operators: spatial isomorphism, unitary equivalence, and similarity, Indiana Univ. Math. J. 59 (2010), 595-620.

[6] R. B. CRofoot, Multipliers between invariant subspaces of the backward shift, Pacific J. Math. 166 (1994), 225-246.

[7] P. A. Fuhrmann, On a class of finite dimensional contractive perturbations of restricted shift of finite multiplicity, Israel J. Math. 16 (1973), 162-175.

[8] S. R. Garcia And M. Putinar, Complex symmetric operators and applications, Trans. Amer. Math. Soc. 358 (2006), 1285-1315.

[9] R. Khan, Dan. Timotin, Matrix valued truncated Toeplitz operators: Basic Properties, Journal of Complex Analysis and Oper. Theory, 2017, doi:10.1007/s11785-017-0675-3.

[10] V. V. Peller, Hankel Operators and their Applications, Springer Verlag, New York, 2003.

[11] D. SARASON, Algebraic properties of truncated Toeplitz operators, Oper. Matrices 1 (2007), 491-526.

[12] B. Sz.-NAGy, C. Foias, H. Bercovici, L. KérChy, Harmonic Analysis of Operators on Hilbert Space, Revised and enlarged edition, Universitext, Springer, New York, 2010. 